\title{
THE NON-FOURIER HEAT CONDUCTION MODEL BETWEEN THE CASTING ROLLER AND THE SLAB
}

\author{
Xiong Yonggang ${ }^{1}$, Liu Yunyư ${ }^{2}$, Wu Jiping ${ }^{1}$, Tian Wanpeng ${ }^{1}$, Du Minxian ${ }^{1}$, Tan \\ Jianping ${ }^{3}$ \\ ${ }^{1}$ School of Mechanical Engineering, Hunan University of Technology, Zhuzhou Hunan 412007, \\ China \\ ${ }^{2}$ Department of Mechanical Engineering, Henan Polytechnic Institute Nanyang Henan 473009, \\ China \\ ${ }^{3}$ College of Mechanical and Electrical Engineering, Central South University Changsha Hunan \\ 410083, China \\ a829129393@qq.com
}

Keywords: Material casting, rolling forming, rapid solidification.

\begin{abstract}
Material casting and rolling forming technology is a new technology of metal rapid solidification of near net and near final forming. The high gradient temperature at the interface is a remarkable characteristic of the interface heat transfer. The heat conduction between the roller and the slab is a heat transfer behavior with dynamic boundary and directional reinforcement. Consider non Fourier heat conduction effect and analysis heat conduction mechanism and characteristics of the cast roll to determine its boundary conditions, established the non Fourier heat conduction model between the Cast roll and the slab, laying the foundation for calculate and analyse the optimal process parameters and cooperative model's establishment.
\end{abstract}

\section{Introduction}

Twin-roll continuous casting is a nearly eventually forming technology, which directly to make the liquid metal into semi-finished or finished products, it will be the traditional production process of liquid metal ingot casting and hot rolling plate strip multichannel independent process such as highly concentrated on a roll casting device for completion, which including two different processes of "casting" and "hot rolling", that is a "high cooling rate, continuous large deformation" production process $^{[1-10]}$, which can short process, high efficiency product sheet metal strip, and the production process is shown in figure 1.

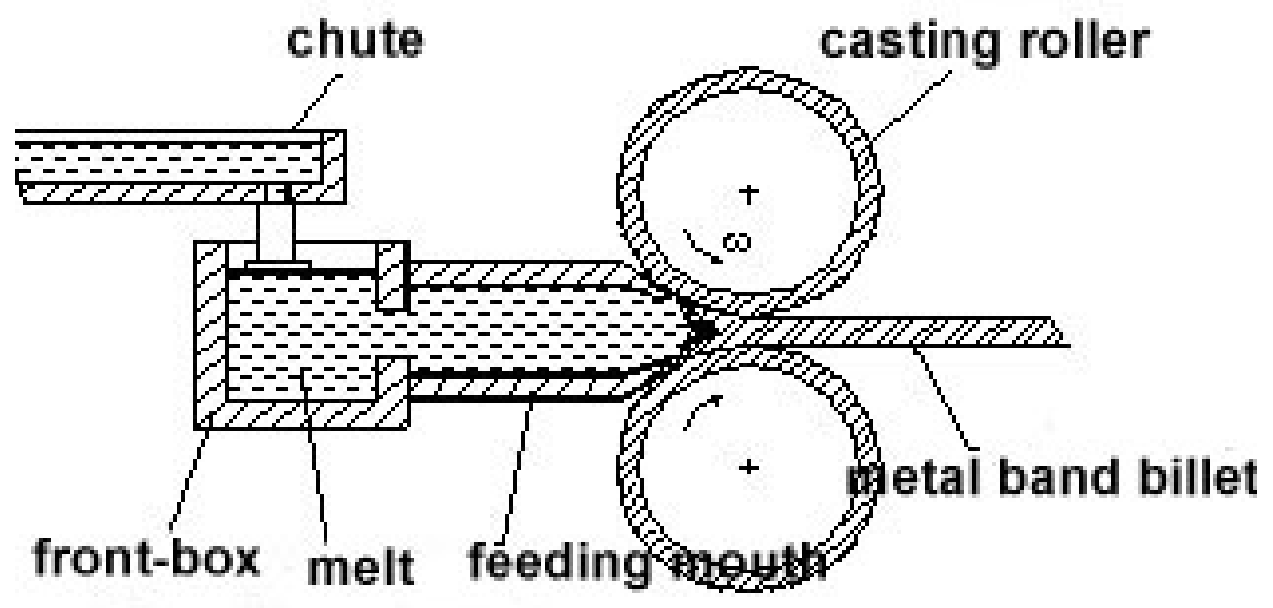

Fig.1 Process diagram of the aluminium slab and the casting roller

Material roll technology is a kind of near net shape and nearly eventually forming new technology ${ }^{[1-2]}$, it used materials in the process of transition from semi-solid liquid solid shown 
excellent mobility and low deformation resistance, to realize the material processing molding, therefore, roll casting technology belongs to the high efficient short process technology ${ }^{[2-3]}$. In the process of aluminum roll forming, the high temperature liquid aluminum through the only a few millimeters forming zone fastly, should complete the solidification cooling molding process of the material's casting and rolling process, therefore, the high gradient temperature on forming interface is the interface heat transfer's marked characteristics. The high temperature gradient of the forming interface has two aspects of meaning ${ }^{[3-5]}$ : on the one hand, because of the high gradient temperature between the roller shell and the aluminum contact interface, in order to maintaining the high heat flow density on forming interface, meet the requirements of forming process on the system heat transfer ability. On the other hand, because of the high gradient temperature of the aluminous material itself on the forming interface, in orfer to meet the requirements of the production process of aluminum solidification cooling rate and the outlet temperature, to ensure product quality and production efficiency.

\section{HEAT CONDUCTION MECHANISM AND CHARACTERISTICS BETWEEN THE SLAB AND THE CASTING ROLLER}

Aluminum roll forming process is shown as figure 2, the molten liquid aluminum through the nozzle into the wedge area between the two rotating casting roller, in the length of about $70 \mathrm{~mm}$ cast-rolling area, metallic melts after casting and rolling directly produce different thickness of the strip $^{[4]}$. In the process of roll casting, aluminum solidification and cooling release a great deal of latent and sensible heat, this heat is mainly through the interface between casting roller and molding contact passed to the roll ring, then by the roll ring internal circulation cooling water cooling roller set constantly and take away the heat, to ensure the roll casting process to continue ${ }^{[5]}$. Therefore, compare to rolling technology, roll forming process has 3 prominent characteristics ${ }^{[6]}$ : 1)The casting and rolling of the metal melt completed one time, have the solid-liquid phase change and strong cold process of the metal, in material molding process will release a lot of heat. 2)Casting roller is different from ordinary roller mechanism, which have dual function of the water-cooled mould and the rolling thin strip. 3) Roll casting process is done under the high temperature drop strong outfield. Therefore, Large temperature gradient and strong cooling speed is the most significant features of roll forming process, the heat transfer problem on the forming interface is the most important and critical problem in the system.

The process of aluminum cast-rolling's interface heat transfer is shown as figure 2 . In the location of the cast-rolling's entrance, the molten metal in contact with the outside surface of the cold roll ring, the roller set's heating temperature began to rise, liquid aluminum start to cooling and solidification, through the casting area and rolling area, aluminium strip separates the roller set in the location of the export, out of the roll casting zone. The outer surface of the roller sleeve close to the cast rolling zone is close to the high temperature aluminum liquid or aluminum strip, the roller set continually by the aluminium strip's radiation heating, the rest of the position of roll surface by natural convection air cooling, the internal surface of the roller set always affected by internal circulating water cooling.

In the cast rolling zone, the roller surface temperature significantly reduced under the condition of rapid cast-rolling, but in $98 \%$ of the roller shell surface roll casting zone, the surface temperature of the roller set rise generally, for aluminium strip, because the molding speed increase, making the aluminum slab temperature gradient further increase, aluminium slab surface's temperature gradient in the middle of the peak value of $-1180^{\circ} \mathrm{C} / \mathrm{s}$, increase $45 \%$, the average temperature gradient is $-645^{\circ} \mathrm{C} / \mathrm{s}$, increase $29 \%$, therefore aluminum strip roll casting is a metal rapid solidification, with the increase of roll casting speed, the cooling intensity up to the orders of magnitude with $10^{6} \mathrm{~K} / \mathrm{s}$.

But many studies ${ }^{[6-9]}$ regard the rapid heat transfer process as a general Fourier heat conduction, the study also showed that, at room temperature, the range of $\tau$ is about $10^{-14} \mathrm{~s} \sim 10^{-11} \mathrm{~s}$ to metal, about $10^{-8} \mathrm{~s} \sim 10^{-10} \mathrm{~s}$ to gas, but to liquid and insulation, which between the two values. under low temperature, the range of $\tau$ is about $10^{-11} \mathrm{~s} \sim 10^{-6} \mathrm{~s}$ to metal. For the porous medium(for example sand), biological tissu(meat), their thermal relaxation time is higher, which can reach dozens of seconds at 
room temperature ${ }^{[10-13]}$. That is to say, except the biological tissue and porous media, the thermal relaxation time $\tau$ of the engineering material is from nanosecond to picosecond. Which indicate if the physical process happens in microseconds or larger time interval, these materials's non Fourier effect ${ }^{[10,14-15]}$ in unsteady heat conduction will be very important.

In conclusion, aluminum slab casting roller's heat conduction has the following characteristics: it is a kind of metal rapid solidification, has dynamic boundary, from aluminium slab to casting roller and roller core(see fig.2), by directional thermal conductivity, which is strengthening heat transfer. Therefore, this paper consider non Fourier effect on the influence of aluminium slab cast-rolling, establish the non Fourier heat conduction model between the casting roller and the aluminium slab.
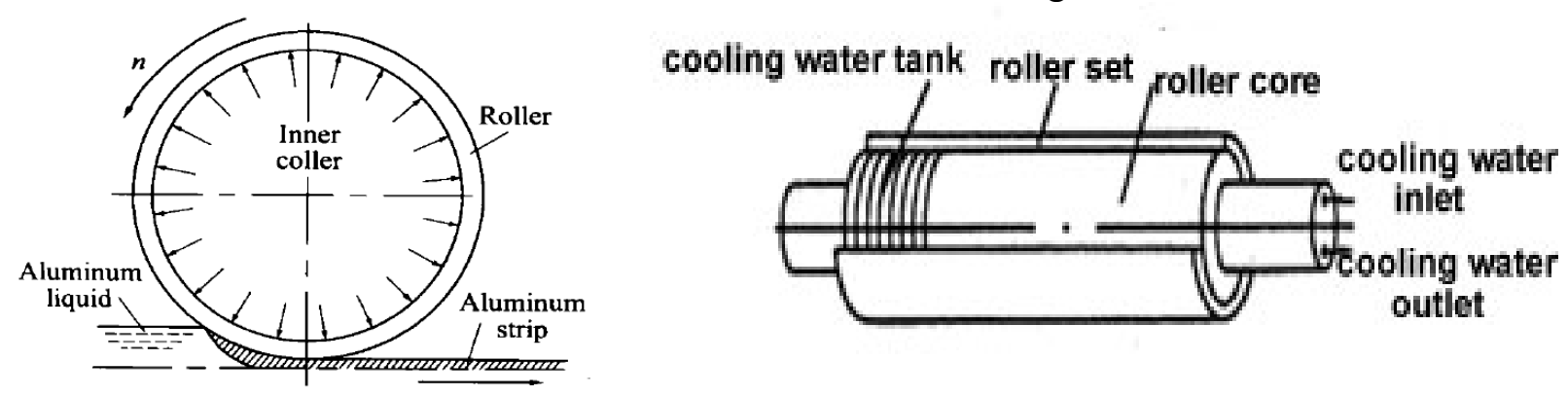

Fig.2 Interface heat exchange process of cast-rolling shaping

\section{HEAT CONDUCTION MODEL BETWEEN THE CASTING ROLLER AND THE SLAB BASED ON NON-FOURIER EFFECT}

\section{Basic Equation of Heat Transfer}

Heat exchange process of aluminium rolling treat the central plane of the strip as symmetry plane, therefore, it should only study the upper part of the heat transfer process. As shown in figure 2. In order to get the differential equation ${ }^{[8]}$ of heat transfer system, firstly should determine the appropriate coordinate system, to simple the finite sequence solution.

According to the research object, for casting roller set, using polar coordinate system, and place the coordinates in the space occupied by the roll cover, which not movement with roller set, fix the polar coordinate origin in the center of the roll, and let the $\gamma$ axial direction in accordance to casting roller radial, $\phi$ is the roll angle direction. For aluminum strip, use rectangular coordinate system, the coordinates origin is placed on the symmetry plane of the entrance of the cast rolling zone, axis $x$ along the rolling direction, axis $y$ along the thickness direction. Due to use static coordinate system, so for the control micro unit, except the heat flux of heat conduction and the calorific value of internal heat source, there is also the quantity of heat through the control unit interface, which is due to the mass flow of the macro motion of the roller set(along $\phi$ direction) and the aluminum strip(along $x, y$ direction $)^{[15-18]}$.

For roller set, no internal heat source, no phase change, the partial differential equations of the two-dimensional unsteady heat conduction is:

$$
\frac{\partial T_{g}}{\partial t}=a\left[\frac{\partial^{2} T_{g}}{\partial t^{2}}+\frac{1}{r} \frac{\partial T_{g}}{\partial t}+\frac{1}{r^{2}} \frac{\partial^{2} T_{g}}{\partial \varphi^{2}}\right]+\varpi \frac{\partial T_{g}}{\partial \varphi}
$$

For aluminium strip, phase change occurs in the process of solidification, will release a large amount of crystallization latent heat, here regard the crystallization latent heat as internal heat source, then the partial differential equations of the two-dimensional unsteady heat conduction is:

$$
\begin{gathered}
\frac{\partial T_{b}}{\partial t}=a\left[\frac{\partial^{2} T_{b}}{\partial x^{2}}+\frac{\partial^{2} T_{b}}{\partial y^{2}}\right]+\left[u \frac{\partial T_{b}}{\partial x}+v \frac{\partial T_{b}}{\partial y}\right]+a \frac{q_{v}}{\lambda} \\
q\left(x, t+\tau_{q}\right)=-\lambda \frac{\partial T}{\partial x}\left(x, t+\tau_{T}\right)
\end{gathered}
$$




$$
\tau_{q} \frac{\partial^{2} T}{\partial t^{2}}+\frac{\partial T}{\partial t}=\alpha \frac{\partial^{2} T}{\partial x^{2}}+\tau_{T} \alpha \frac{\partial^{3} T}{\partial x^{2} \partial t}
$$

Here, $\tau_{q}$ and $\tau_{T}$ indicate heat flow relaxed time and temperature relaxed time respectively. Which can adjust different relaxation time to apply to thermal wave, thermal diffusion, electronic-phonon two-step thermal conductivity and other situations. In the formula, $T_{g}$-Roller set temperature, $T_{b}$ - strip temperature, $t$-time, $\varpi$ - Roller rotation speed, $u, v-$ Velocity component of the control micro unit along $x, y$ direction, $a$ - Thermal diffusivity of the Roll material, $\frac{\partial T}{\partial t}$ is local rate of change of temperature, which value is 0 under steady state condition, $\varpi \frac{\partial T_{g}}{\partial \varphi}$ and $u \frac{\partial T_{b}}{\partial x}+v \frac{\partial T_{b}}{\partial y}$ are partial differential equation of thermal conductivity reflects the heat transfer characteristics in the process of roll casting, that is except the micro particle thermal motion caused by heat conduction, there is also energy transfer caused by object displacement, is that the combination of the two basic modes of heat transfer.

\section{Boundary Conditions}

1) Rolling interface is the third kind boundary condition

$$
q=h_{j}\left(T_{b}-T_{g}\right)
$$

In the equation: $q$ - heat flow density, $h_{j}$ - heat transfer coefficient of the roll and the strip contact interface $^{[21-22]}$.

2) In the zone of non-cast rolling. The calculation of the roll cover, the contact place of the strip and the air is carried out by convection heat transfer and radiant heat transfer.

3) The heat exchange between the roll cooling sink and cooling water adopt turbulent convection heat transfer model:

$$
q=h_{\sigma}\left(T_{g}-T_{w}\right)
$$

In the equation: $T_{w}$ is cooling water inlet temperature, $h_{\sigma}$ is the heat transfer coefficient between cooling water tank and cooling water, $h_{\sigma}$ can calculate by the equation below:

$$
\frac{h_{\sigma} \circ D}{K_{\sigma}}=0.023 \circ\left[\frac{D \circ V_{\varpi}}{\eta}\right]^{0.8}\left[\frac{c_{\varpi} \circ \eta}{K_{\sigma}}\right]^{0.4}
$$

In the equation: $D$ is the equivalent diameter of the cooling water tank, $K_{\sigma}$ is the heat conductivity coefficient of cooling water, $\eta$ is the viscosity of water, $V_{\sigma}$ is the flow of water, $c_{\varpi}$ is the specific heat of water.

4) In the section $Z=0$, which can be considered as adiabatic, that is $\frac{\partial T}{\partial Z}=0$ when $Z=0$.

\section{Summary}

Analysis and introduce the rolling aluminum sheet process and the thermal conductivity characteristic between roll and slab, consider the non Fourier heat conduction effect, and established the thermal conductivity model between the roll and slab, which laid a solid foundation for calculating heat conduction between roll and slab. 


\section{Acknowledgement}

This researche is support by NCFS ( 51345005), NSFC of Hunan province (13JJ9014), Jiangsu Province Postdoctoral Science Foundation (1202003B)). National key basic research project (G1999064906)

\section{References}

[1] Zhong Jue. Progress of basic research to improve the quality of aluminum[J]. Light Alloy Fabrication Technology, 2002, 30(5):1 -10.

[2] Zhong Jue. The basic research of material preparation machinery under the exceptional physical field condition[J]. Chinese Journal of Mechanical Engineering, 2000, 11(2):12 -20.

[3] Cortes. The new demands of thin strips casting[J]. Light Metals, 1995, 1161-1164.

[4] Cui Xiaochao, Wang Youhong, Liu Cai. Quasi flow function method analysis the flow condition of the rolling process[J]. Chinese Journal of Nonferrous Metals, 2002, 156-162.

[5] Gao Zhi. The heat transfer mechanism of material[liquid-solid]rheologic shaped interface and the estab-lishment of shaped interface based on the high grad of change of temperature[D]. Changsha: Central South University, 2001.

[6] Shi Rong, Cui Xiaochao, Sun Binyu. Numerical simulation for solidification of aluminium-strip in continuous casting and rolling process[J]. The Chinese Journal of Nonferrous Metals, 1996, 6(2):98 -102.

[7] Gao Zhi, Xiao Gang, Zhong Jue. The mechanismresearch of external cooling in aluminum cast-rolling pro-cess[J]. Journal of Central South University of Technology, 2001, 32(2):192195.

[8] Li Xiaoqian. A mathmatic model for temperature field ofcontinuous roll casting sheet in rolling zone[J]. The Chinese Journal of Nonferrous Metals, 1997, 7(4):168-170.

[9] Daaland O, Espedal A B, Nedreberg M L, et al. Thingauge twinroll cast-rolling process capabilities and productquality[J]. Light Metals, 1997, 745- 752.

[10] Gao Zhi, Xiao Gang. Simulation of heat transfer behavior of shaped interface in aluminum cast-rolling process[J]. The Chinese Journal of Nonferrous Metals, 2003, 13(6):1350-1357.

[11]Zhang Zhe, Liu Dengying. The research progress of the non Fourier heat conduction[J]. Advances in Mechanics, 2000, 30(3):123-141.

[12] Maxwell J C. On the dynamic theory of gases. Philosophics Transactions Loodon, 1967, 157:49-85.

[13] Cattaneo C. A form of heat conduction equation which eliminates the paradox of instantaneous propagation Compute Rendus, 1958, 247:431-433.

[14] Morse P M, Feshbach H. Methods of Theoretical Physics. NewYork: McGraw-Hill, 1953.

[15] Vernotte P. Some Possible complications in the phenomena of thermal conduction. Compute Rendus, 1961, 252:2190-2191.

[16]Chester M. Second sound in solid. Physical Review, 1963, 131:2013-2016.

[17] Nettleton R E. Relaxation theory of thermal conduction in liquids. Physics of Fluids, 1960, 3:216-225.

[18] Maurer M J. Relaxation model of heat conduction in metal. Journal of Applied Physic, 1969, 40:5123-5127.

[19]Francis H P. thermo-mechanical effects in elastic wave propagation:Asurvey. Journal of Sound and Vibration, 1972, 21:181-192. 
[20] Wang Haidong, Liu Jinhui, Guo Zengyuan, Gao Qiaohoushi. In the steady state of metal nanofilms non Fourier heat conduction experiments[J]. Chinese Science Bulletin, 2012, 59(19):1794-1799.

[21] Sun Xin, Cheng Xinguang, Meng Ji'an, Guo Zengyuan. Validation of laminar flow field coordination equation and its properties[J]. Journal of Engineering Thermophysics, 2005, 26(2).

[22] Karwe M V. Jaluria Y. Numerical simulation of thermal transport associate with a continuously moving flat sheet in materials processing[J]. Transaction of the ASME, 1991, 113:612-619. 